\title{
AVALIAÇÃO DO EQUILIBRIO \\ E DO CONDICIONAMENTO \\ CARDIORRESPIRATÓRIO DE \\ PARTICIPANTES DO GRUPO DE ATIVIDADES \\ HIDROCINESIOTERAPEUTICAS DO CENTRO \\ UNIVERSITÁRIO FRANCISCANO \\ EM SANTA MARIA, RS
}

Luana Rossato Siqueira Motta

Alexandre Rodrigues Seero²

Érika Corrêa Machado ${ }^{3}$

Natália Ferraz Mello ${ }^{4}$

Nadiesca Taísa Filippin 5

Rafael Corrêa Gobbato ${ }^{6}$

Alecsandra Pinheiro Vendrusculo?

1 Acadêmica de Fisioterapia do Centro Universitário Franciscano. E-mail: luanaa_motta@hotmail.com.

2 Graduado em Fisioterapia pelo Centro Universitário Franciscano. E-mail: alex.rsevero@hotmail.com.

3 Graduada em Fisioterapia pelo Centro Universitário Franciscano.

E-mail: erika.c.machado@hotmail.com.

4 Acadêmica de Fisioterapia do Centro Universitário Franciscano. E-mail: taia_mello@hotmail.com.

5 Graduada em Fisioterapia. Mestre e Doutora em Fisioterapia. Professora Associada do Centro Universitário Franciscano. E-mail: nadi.filippin@yahoo.com.br.

6 Graduado em Fisioterapia. Mestre do programa de Pós-Graduação em Cirurgia Plástica.

E-mail: rafagobbato@hotmail.com.

7 Graduada em Fisioterapia. Mestre em Ciências do Movimento Humano. Professora Associada do Centro Universitário Franciscano. E-mail: alec@unifra.br. 
O envelhecimento associado ao sedentarismo gera mudanças nos sistemas neuromuscular, somatossensorial, vestibular e visual. $\bigcirc$ exercício físico é importante na manutenção da funcionalidade dessa população, e a fisioterapia aquática (FA) é uma opção segura e viável. Esta consiste em exercícios realizados em piscina aquecida que promovam alterações fisiológicas nos diversos sistemas do corpo, facilitando a realização de exercícios. $\bigcirc$ presente estudo avalia o equilíbrio e o condicionamento cardiorrespiratório de participantes do Grupo de Atividades Hidrocinesioterapêuticas. A amostra foi composta por 23 idosos que realizaram FA uma vez por semana, com duração de 50 minutos, durante 8 semanas. A sessão foi dividida em 4 etapas: 10 minutos de aquecimento, 20 minutos de fortalecimento em geral, exercícios de equilíbrio e coordenação, e 10 minutos de alongamento e 10 minutos de relaxamento. Foram utilizados para a avaliação do equilíbrio a Escala de Equilíbrio de Berg (EEB) e o Teste de Caminhada de Seis Minutos (TC6) para a avaliação do condicionamento cardiorrespiratório. As avaliações foram realizadas em três momentos: no início do programa de FA (Pré), 4 semanas após (Pós 1) e 8 semanas após (Pós 2). Verificou-se diferença estatisticamente significativa nos períodos, tanto na avaliação do equilíbrio como no condicionamento cardiorrespiratório, ou seja, ao longo do programa de FA, os participantes aumentaram a distância percorrida no TC6 e realizaram as tarefas da EEB com maior facilidade. Conclui-se que a FA tem um papel importante na manutenção do equilíbrio e do condicionamento cardiorrespiratório de idosos, devendo sua prática ser estimulada nessa população.

palavras-chave

Idosos. Equilíbrio. Condicionamento Cardiorrespiratório. Fisioterapia Aquática.

A proporção de idosos no Brasil hoje compõe um contingente acima de 22 milhões de pessoas, superando a população de vários países europeus como a França, a Inglaterra e a Itália, de acordo com estimativas das Nações Unidas (SACHETTI et al., 2012). Especificamente, o processo de envelhecimento é acompanhado de mudanças nos sistemas neuromuscular, somatossensorial, 
vestibular e visual e está associado a um estilo de vida sedentário (ALFIERI; MORAES, 2008; MARTIN; SHEAFF, 2007). Isso se torna particularmente importante diante do aumento da proporção de idosos na população mundial e, em especial, no Brasil. A formação de prejuízo físico, psicológico e social resulta em altos custos com os cuidados de saúde, um problema de saúde pública (ALFIERI et al., 2012; SHUBERT, 2011; CLOSE et al., 1999). O exercício físico pode ser um aliado na redução de prejuízos do processo de envelhecimento por diminuir a perda óssea e a massa muscular, reduzindo o risco de fraturas em até 60\% (STEVENS; OLSON, 2000; GREGG et al., 2000).

A hidroterapia em grupo consolida-se como uma alternativa para a prática de exercícios físicos e tem sido utilizada como um recurso para tratar doenças reumáticas, ortopédicas e neurológicas (ALIKHAJEH; HOSSEINI; MOGHADDAM, 2012). Sendo um método terapêutico que utiliza os princípios físicos da água em conjunto com a cinesioterapia, é uma opção para prevenir, manter, retardar, melhorar ou tratar as disfunções físicas características do envelhecimento (CAROMANO; CANDELORO, 2001; CANDELORO; SILVA, 2000). O meio aquático submete o organismo a diferentes forças físicas e, em consequência, realiza uma série de adaptações fisiológicas, além de possuir vantagens para esse grupo populacional. Com o aproveitamento das suas propriedades, esse ambiente possibilita um melhor rendimento aos idosos por ser considerado seguro e eficiente para a reabilitação (CANDELORO; CAROMANO, 2007; ALVES et al., 2004; CAROMANO; FILHO; CANDELORO, 2003). Nos exercícios aquáticos, a sobrecarga articular é menor, diminuindo assim o risco de quedas e lesões. Além disso, a flutuação permite ao indivíduo realizar exercícios e movimentos que não podem ser feitos no solo (ALIKAJEH; HOSSEINI; MOGHADDAM, 2012).

A fisioterapia aquática (FA) em grupo trata-se de um recurso utilizado pelo fisioterapeuta que assiste e acompanha os grupos a partir de objetivos estabelecidos para cada um deles, com o intuito de reabilitar e/ou prevenir distúrbios e diversas patologias. Atualmente, a FA, segundo Ferreira (2010), proporciona a socialização e maior motivação e concentração.

Diante do exposto acima, justifica-se o trabalho devido ao conhecimento de que a população idosa cresce significativamente e tem a sua funcionalidade alterada e é uma parcela da população que a fisioterapia atua tanto em grupo como individualmente. Por isso, se faz necessário aprofundar o conhecimento técnico-científico sobre os benefícios da FA na promoção e na reabilitação da saúde. Baseado nesta premissa, o objetivo da presente pesquisa foi avaliar o equilíbrio e o condicionamento cardiorrespiratório de participantes do Grupo de Atividades Hidrocinesioterapêuticas do Centro Universitário Franciscano em Santa Maria, RS. 
Trata-se de uma pesquisa de caráter quantitativo e do tipo quase-experimental com pré-teste, teste intermediário e pós-teste. A amostra foi constituída por 23 indivíduos idosos, sendo 2 do gênero masculino e 21 do gênero feminino, residentes no município de Santa Maria, RS.

Os critérios de inclusão desta pesquisa foram idade entre 60 e 80 anos, indivíduos com o cognitivo preservado, independentes funcionais e com encaminhamento médico para a realização da FA. Os critérios de exclusão desta pesquisa foram idade inferior a 60 anos e superior a 80 anos, idosos que apresentassem défice de compreensão para entender os comandos solicitados, que apresentassem insuficiência cardíaca congestiva, pressão arterial descompensada (sem o uso de medicação controlada), acidente vascular encefálico (AVE) prévio, $25 \%$ de faltas durante o período das práticas realizadas na piscina e que não aceitassem participar da pesquisa. Após o aceite de pesquisa, a amostra assinou o Termo de Consentimento Livre e Esclarecido, o qual foi devidamente lido e explicado pelos pesquisadores.

Os participantes do estudo realizaram FA uma vez por semana, com duração de 50 minutos, durante 8 semanas. A sessão era dividida em 4 etapas: 10 minutos de aquecimento, 20 minutos de fortalecimento em geral, exercícios de equilíbrio e coordenação, 10 minutos de alongamento e 10 minutos de relaxamento. Os exercícios propostos em cada etapa foram embasados na literatura atual, como Koury (2000), Sacchelli, Accacio e Radl (2007) e Ruoti, Morris e Cole (2000).

Os dados obtidos para a pesquisa foram coletados a partir de uma ficha de avaliação contendo os dados pessoais, a anamnese do usuário e as pontuações da Escala de Equilíbrio de Berg (EEB) e do Teste de Caminhada de Seis Minutos (TC6). As avaliações foram agendadas em horários predeterminados pelos pesquisadores.

Para a mensuração do equilíbrio, foi utilizada a Escala de Equilíbrio de Berg (EEB), criada em 1992 por Katherine Berg, traduzida e adaptada para a língua portuguesa por Miyamoto e considerada um instrumento confiável para ser usado na avaliação do equilíbrio dos pacientes idosos. A EEB é um instrumento validado, de avaliação funcional do equilíbrio composta de 14 tarefas com cinco itens cada e pontuação de 0-4 para cada tarefa: 0- é incapaz de realizar a tarefa e 4- realiza a tarefa independentemente. $\mathrm{O}$ escore total varia de $0-56$ pontos: 
quanto menor for a pontuação, maior é o risco para quedas; quanto maior, melhor o desempenho (GAZZOLA et al., 2006; CHRISTOFOLETTI et al., 2006).

Para as aplicações da EEB, os pacientes foram agendados em horários predeterminados pelos pesquisadores, e a coleta ocorreu nas dependências do Laboratório de Ensino Prático (LEP) de Fisioterapia do Centro Universitário Franciscano, onde os pacientes foram avaliados pelo mesmo pesquisador.

O Teste de Caminhada de Seis Minutos (TC6) constitui um teste seguro, válido e confiável e requer um mínimo de equipamentos para sua realização (BRITO; SOUZA, 2005). Anterior à aplicação do teste, foi realizado um período de repouso de no mínimo 10 minutos, sendo neste intervalo aferidas a pressão arterial, a oximetria de pulso e a frequência cardíaca e respiratória para a segurança dos participantes durante a realização do teste.

$\mathrm{O}$ teste foi realizado em um corredor com comprimento mínimo de 30 metros e livre de circulação de pessoas, com marcações de metro em metro. Os pacientes foram orientados a caminhar em linha reta durante 6 minutos, mantendo o ritmo de caminhada, respeitando seus limites e sendo acompanhados pelos pesquisadores durante o trajeto. Foi controlada a distância percorrida durante os 6 minutos e o tempo pelo cronômetro Nautika Procron.

A EEB e o TC6 foram avaliados nos mesmos momentos, ou seja, no início do programa de FA (Pré), 4 semanas após (Pós 1) e 8 semanas após (Pós 2). Os dados foram analisados através de estatística descritiva (média e desvio-padrão). Foi aplicado o teste de Friedman e, a seguir, foi realizado o teste de post hoc de Dunn para comparar a diferença entre as avaliações do teste de equilíbrio. Para comparar a diferença entre as avaliações do teste de condicionamento cardiorrespiratório, foi aplicado o teste de post hoc de Bonferroni. $\mathrm{O}$ programa utilizado foi o SPSS 18.0, sendo o nível de significância de $\mathrm{p} \leq 0,05$. O projeto foi submetido e aprovado pelo Comitê de Ética e Pesquisa do Centro Universitário Franciscano, sob o número 300.2010.2.

\section{Resultados}

A amostra foi constituída por 23 indivíduos idosos, com média e desvio padrão de idade de 62,8 $\pm 8,34$ anos, respectivamente. Quando considerados os dados de equilíbrio após a análise estatística, foi verificada diferença estatisticamente significativa nos três momentos avaliados (Pré, Pós 1 e Pós 2), ou seja, houve uma melhora do equilíbrio ao longo do programa de FA (Tabela 1). 
Tabela 1 - Valores da média e desvio padrão dos três momentos de avaliação do equilíbrio (Pré, Pós 1 e Pós 2) e valores do nível de significância após o post hoc de Dunn. P1 (comparação do Pré com Pós 1); P2 (comparação do Pré com Pós 2); P3 (comparação do Pós 1 com Pós 2); EEB (Escala de Equilíbrio de Berg).

\begin{tabular}{|c|c|c|c|c|c|}
\hline EEB & Média & $\begin{array}{l}\text { Desvio } \\
\text { Padrão }\end{array}$ & $\mathrm{P} 1$ & $\mathrm{P} 2$ & P3 \\
\hline Pré & 49,6 & $\pm 2,2$ & \multirow{3}{*}{$P<0,05$} & \multirow{3}{*}{$P<0,01$} & \multirow{3}{*}{$P<0,05$} \\
\hline Pós1 & 53,5 & $\pm 2,63$ & & & \\
\hline Pós2 & 55,5 & $\pm 0,7$ & & & \\
\hline
\end{tabular}

Para comparação da distância percorrida no TC6 nas três avaliações (Pré, Pós 1 e Pós 2), foi utilizado o teste ANOVA One-way de medidas repetidas, o qual apresentou $\mathrm{p}<0,0001$, significando diferença estatística entre as avaliações. Para avaliar a diferença entre as avaliações, realizou-se o teste post hoc de Bonferroni, o qual apresentou os resultados demonstrados na Tabela 2.

Tabela 2 - Valores das distâncias percorrida no TC6 e nível de significância do teste post hoc de Bonferroni entre as avaliações. P1 (comparação do Pré com Pós 1); P2 (comparação do Pré com Pós 2); P3 (comparação do Pós 1 com Pós 2).

\begin{tabular}{|c|c|c|c|c|c|}
\hline TC6 & Média & $\begin{array}{l}\text { Desvio } \\
\text { Padrão }\end{array}$ & $\mathrm{P} 1$ & $\mathrm{P} 2$ & P3 \\
\hline Pré & 180,32 & $\pm 35,5$ & \multirow{3}{*}{$P<0,01$} & \multirow{3}{*}{$P<0,01$} & \multirow{3}{*}{$P<0,01$} \\
\hline Pós1 & 243,88 & $\pm 38,3$ & & & \\
\hline Pós2 & 295,8 & $\pm 63,0$ & & & \\
\hline
\end{tabular}

\section{Discussão}

Foi verificado no presente estudo que a prática da FA contribuiu na melhora do equilíbrio e do condicionamento cardiorrespiratório de indivíduos idosos participantes do Grupo de Atividades Hidrocinesioterapêuticas. O envelhecimento prejudica várias etapas do controle postural, diminuindo a capacidade compensatória e levando a um aumento da instabilidade (MEEREIS; ROSSATO; MOTTA, 2011). A prática de exercício físico pode interferir positivamente na capacidade funcional de idosos, melhorando assim a sua saúde e qualidade de vida, pois a água é um meio diferente e que está associado à atividade prazerosa de relaxamento num ambiente agradável e de fácil socialização (SILVA, 2010; SILVA et al., 2013). Os exercícios físicos visam a melhorar a condição de 
equilíbrio, pois favorecem o aprimoramento das condições de recepção de informações sensoriais do sistema vestibular, visual e somatossensorial e ainda ativam os músculos antigravitacionais, reestruturando por sua vez o equilíbrio (MEEREIS et al., 2013).

É importante ressaltar que a água permite a imersão e a flutuação corporal, facilitando a reprodução de movimentos compostos de maneira segura e variada, minimizando os impactos (quando comparados com os exercícios no solo), o que pode permitir o trabalho de mobilidade corporal e de flexibilidade de forma segura e gradual (BASTOS; OLIVEIRA, 2003). A FA é uma técnica essencial na movimentação corporal e as propriedades físicas da água provocam efeitos como o relaxamento, a analgesia, a redução do impacto e agressões sobre as articulações, ao contrário dos exercícios no solo, que contribuem para o risco de intercorrências (BIASOLI; MACHADO, 2006).

Segundo Bruni, Granado e Prado (2008) pode ser observado uma melhora significativa na força muscular e potência da maioria dos músculos avaliados nos idosos que praticaram hidroterapia, tendo como um fator importante a resistência que a água promove durante a realização dos movimentos. Este estudo corrobora com o que McDermott e Mernitz (2006) discorrem, que o treinamento progressivo previne a perda de massa muscular e força, podendo causar uma melhora nesses aspectos, o que auxilia na aquisição de um melhor controle postural.

Um estudo realizado por Resende, Rassi e Viana (2008) avaliou o efeito de um programa de hidroterapia no equilíbrio e no risco de quedas em idosas, por meio da Escala de Equilíbrio de Berg e Timed Up \& Go. Portanto, constatou que um programa de hidroterapia para equilíbrio de baixa a moderada intensidade composto por uma fase de adaptação ao meio aquático, fase de alongamento e fase de exercícios estáticos e dinâmicos para equilíbrio, com duração de 12 semanas, pode aumentar o equilíbrio e reduzir o risco de quedas nas idosas.

De acordo com os resultados obtidos por meio dos dois testes utilizados no presente estudo, o equilíbrio melhorou de forma estatisticamente significante após a execução do programa de FA proposto, o que também ocorreu com o condicionamento cardiorrespiratório dos idosos. Estes resultados possivelmente ocorreram por consequência das respostas ao exercício físico serem mais evidentes durante as primeiras semanas de tratamento, quando as adaptações neuronais predominam e ocorrem adaptações musculares na fase intermédia (RESENDE; RASSI; VIANA, 2008). Esta explicação pode justificar também outro achado do presente estudo, que foi a melhora nos itens avaliados com a realização de FA uma vez por semana. 
Os resultados expostos neste estudo indicam a existência do efeito benéfico na prática de FA, em que os participantes obtiveram resultados positivos no equilíbrio estático e dinâmico, bem como no condicionamento cardiorrespiratório, respectivamente, segundo a Escala de Equilíbrio de Berg e o Teste de Caminhada de Seis Minutos. Isso demonstra que apesar de o processo de envelhecimento ocasionar declínios no equilíbrio e no condicionamento cardiorrespiratório, com a prática de FA pode-se obter uma melhora nesses aspectos e, por consequência, proporcionar uma melhora na capacidade funcional dos idosos praticantes.

\author{
EVALUATION OF BALANCE \\ AND CARDIORESPIRATORY FITNESS \\ OF A HYDROCINESIOTHERAPEUTIC ACTIVITY \\ GROUP AT CENTRO UNIVERSITÁRIO \\ FRANCISCANO IN SANTA MARIA, RS, BRAZIL
}

\title{
abstract
}

The aging associated with physical inactivity causes changes in neuromuscular and sensory systems. Physical exercise is important in maintaining the functionality of this population and the aquatic therapy (AT) emerges as a safe and viable option. This consists of exercises performed in a heated pool that promote physiological changes in various systems of the body. The present study assessed the balance and cardiorespiratory fitness of participants of an AT group. Twenty-three elderly participated in this study. The AT program was performed 50 minutes per day, one day per week for eight week. Each session consisted of a ten-minute warm-up, 20 minutes of general strengthening, balance and coordination exercises, 10 minutes of stretching and 10 minutes of relaxation. Balance and cardiorespiratory fitness were assessed by the Berg Balance Scale (BBS) and the Test of 6-minute walk, respectively, at baseline, after four (post-1) and eight weeks (post-2). There was improvement in the balance and cardiorespiratory fitness with significant differences among three evaluations. The results showed that the AT play a major role in maintaining the balance and cardiorespiratory fitness of the elderly. In this way, the AT practice should be encouraged in this population.

keywords

Elderly. Balance. Cardiorespiratory Fitness. Aquatic Physiotherapy. 
ALFIERI, Fábio Marcon; MORAES, Maria Cecilia Leite de. Envelhecimento e o controle postural. Saúde Coletiva, São Paulo, v. 4, n. 19, p.30-33, 2008.

ALFIERI, Fábio Marcon et al. Effectiveness of an exercise program on postural control in frail older adults. Clinical Interventions in Aging, Auckland, v. 7, n. 1, p. 593-598, Dec 2012.

ALIKHAJEH, Yaser; HOSSEINI, Seyyed Reza Attarzadeh; MOGHADDAM, Amir. Effects of hydrotherapy in static and dynamic balance among elderly men. Procedia - Social and Behavioral Sciences, New York, v. 46, n. 1, p. 2220-2224, Dec. 2012.

ALVES, Roseane Victor et al. Aptidão física relacionada à saúde de idosos: influência da hidroginástica. Revista Brasileira de Medicina do Esporte, São Paulo, v. 10, n. 1, p. 31-37, jan./fev. 2004.

BASTOS, Carina Corrêa; OLIVEIRA, Ediléa Monteiro de. Síndrome da fibromialgia: tratamento em piscina aquecida. Lato \& Sensu, Belém, v. 4, n. 1, p. 3-5, out. 2003.

BIASOLI, Maria Cristina; MACHADO, Christiane Márcia Cassiano. Hidroterapia: aplicabilidades clínicas. Revista Brasileira de Educação Médica, Rio de Janeiro, v. 63, n. 5, p. 225-237, maio 2006.

BRITO, Fausto; SOUZA, Joseane de. Expansão urbana nas grandes metrópoles o significado das migrações intrametropolitanas e da mobilidade pendular na reprodução da pobreza. São Paulo em Perspectiva, São Paulo, v. 19, n. 4, p. 48-63, out./dez. 2005.

CANDELORO, Juliana Monteiro; CAROMANO, Fátima A. Efeito de um programa de hidroterapia na flexibilidade e na força muscular de idosas. Revista Brasileira de Fisioterapia, São Carlos, v. 11, n. 4, p. 303-309, jul./ago. 2007.

CANDELORO, Juliana Monteiro; SILVA, Rodrigo Renato da. Proposta de protocolo hidroterapêutico para fraturas de fêmur na terceira idade. 2000. 10 f. Monografia (Pós-Graduação em Hidroterapia) - Universidade Bandeirante de São Paulo, São Paulo, 2000. Disponível em: <http://www.sld.cu/galerias/pdf/sitios/rehabilitacion-bal/ candelorojm_silvarr.pdf>Acesso em: 2 out. 2015.

CAROMANO, Fátima A.; CANDELORO, Juliana Monteiro. Fundamentos da hidroterapia para idosos. Arquivos de Ciências da Saúde da UNIPAR, Umuarama, v. 5, n. 2, p. 187-195, maio/ago. 2001.

CHRISTOFOLETTI, Gustavo et al. Risco de quedas em idosos com doença de Parkinson e demência de Alzheimer: um estudo transversal. Revista Brasileira de Fisioterapia, São Carlos, v. 10, n. 4, p. 429-433, out./dez. 2006.

CLOSE, Jacqueline et al. Prevention of falls in the elderly trial (PROFET): a randomised controlled trial. Lancet, London, v. 353, n. 9147, p. 93-97, Jan. 1999.

FERREIRA, Ana Raquel Gomes. Promoção do envelhecimento activo através da hidroterapia. 2010. 96 f. Monografia Final de Curso (Licenciatura em Fisioterapia) - Escola Superior de Saúde Atlântica, Universidade Atlântica, Barcarena, 2010.

GAZZOLA, Juliana Maria et al. Fatores associados ao equilíbrio funcional em idosos com disfunção vestibular crônica. Revista Brasileira de Otorrinolaringologia, Rio de Janeiro, v. 72, n. 5, p. 683-690, set./out. 2006.

GREGG, Michael D. et al. Discovery of a Classic FR-II Broad Absorption Line Quasar from the FIRST Survey. The Astrophysical Journal, v. 544, n. 1, p.142-149, Nov. 2000.

KOURY, Joanne M. Programa de fisioterapia aquática: um guia para a reabilitação ortopédica. São Paulo: Manole, 2000. 297 p. 
MARTIN, Joanne E.; SHEAFF, Matthew T. The pathology of ageing: concepts and mechanisms. Journal of Pathology, Edinburgh, v. 211, n. 2, p. 111-113, Jan. 2007.

McDERMOTT, Ann Yelmokas: MERNITZ, Heather. Exercise and older patients: prescribing guidelines. American Family Physician, Kansas City, MO, v. 74, n. 3, p. 437-444, Aug. 2006

MEEREIS, Estele Caroline Welter; ROSSATO, Carla Emilia; MOTA, Carlos Bolli. Equilíbrio postural e envelhecimento. Atividade Física, Lazer \& Qualidade de Vida: Revista de Educação Física, Manaus, v. 2, n. 2, p. 59-65, jul./dez. 2011.

MEEREIS, Estele Caroline Welter et al. Influência da hidrocinesioterapia no equilíbrio postural de idosas institucionalizadas. Motriz, Rio Claro, v. 19, n. 2, p. 269-277, abr./ jun. 2013.

RESENDE, Selma Mendes; RASSI, Claudia Maria, VIANA, Fabiana Pavan. Effects of hydrotherapy in balance and prevention of falls among elderly women. Revista Brasileira de Fisioterapia, São Carlos, v. 12, n. 1, p. 57-63, jan.ffev. 2008.

RUOTI, Richard G:; MORRIS, David M.; COLE, Andrew J. Reabilitação aquática. Tradução: Nelson Gomes de Oliveira. São Paulo: Manole, 2000. 463 p.

SACCHELLI, Tatiana; ACCACIO, Letícia Maria Pires; RADL, André Luis Maierá. Fisioterapia aquática. Barueri: Manole, 2007. (Manuais de Fisioterapia).

SACHETTI, Amanda et al. Equilíbrio x envelhecimento humano: um desafio para a fisioterapia. Revista de Ciências Médicas e Biológicas, Salvador, v. 11, n. 1, p. 64-69, jan./abr. 2012.

SHUBERT, Tiffany E. Evidence-based exercise prescription for balance and falls prevention: a current review of the literature. Journal of Geriatric Physical Therapy, La Crosse, WI, v. 34, n. 3, p. 100-108, July/Sept. 2011.

SILVA, Dayanne Teodoro da; CAMARGO, Lívia Robles de. A influência da atividade física na capacidade funcional de idosas. In: MOSTRA ACADÊMICA UNIMEP, 8., 2010, Piracicaba. Anais... Piracicaba: UNIMEP, 2010, p. 1-5.

SILVA, Douglas Monteiro da et al. Efeitos da fisioterapia aquática na qualidade de vida de sujeitos com doença de Parkinson. Fisioterapia e Pesquisa, São Paulo, v. 20, n. 1, p. 17-23, mar. 2013.

STEVENS, Judy A.; OLSON, Sarah. Reducing falls and resulting hip fractures among older women. Morbidity and Mortality Weekly Report, Atlanta, GA, v. 49, n. RR-2, p. 3-12, Mar. 2000

Recebido: 13/05/2014

Aceite Final: 19/11/2015 